
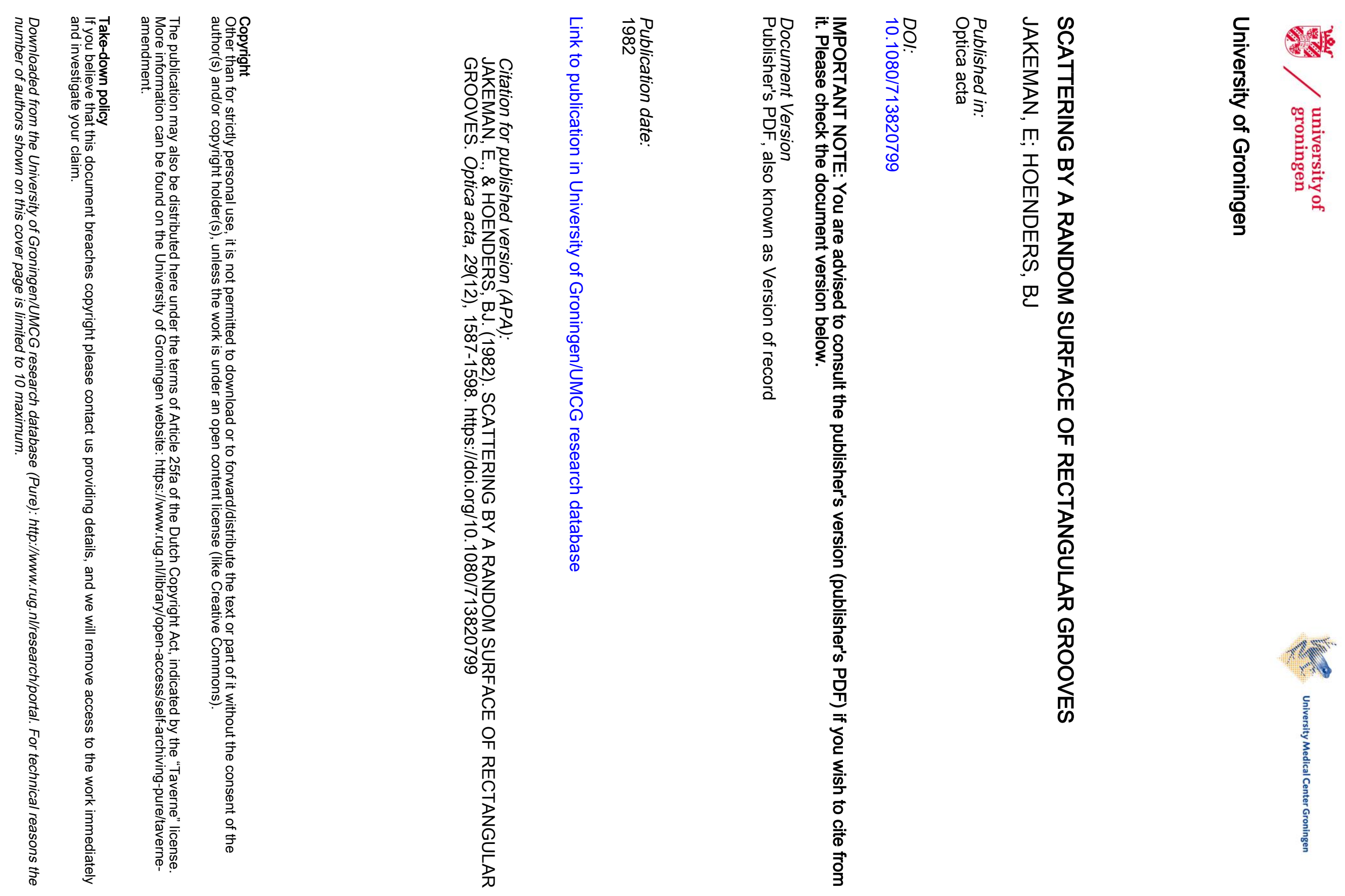
OPTICA ACTA, 1982, VOI. 29, NO. 12, 1587-1598

\title{
Scattering by a random surface of rectangular grooves
}

\author{
E. JAKEMAN
}

Royal Signals and Radar Establishment, Malvern, Worcestershire, England

and B. J. HOENDERS

Department of Applied Physics, University of Gröningen, The Netherlands

(Received 23 March 1982; revision received 17 May 1982)

\begin{abstract}
The results of a preliminary theoretical investigation of the statistical properties of electromagnetic radiation scattered by a randomly grooved surface are presented. The contrast of the intensity pattern is calculated for both nearand far-field geometries, and it is shown that the full probability density of intensity fluctuations can be obtained exactly in the far-field specular direction.
\end{abstract}

\section{Introduction}

It is now well known that when radiation is scattered by a comparatively small region of a scattering medium, enhanced non-gaussian intensity fluctuations may be generated [1]. These are of considerable interest as they not only contain more information than the usual gaussian fluctuations (speckle) but may also provide a more severe noise limitation on system performance. Consequently a good deal of theoretical and experimental attention has been devoted to scattering configurations which can generate non-gaussian noise [2]. The theoretical model which has perhaps been most thoroughly investigated in this context is the random phase-changing screen: a 'thin' scattering layer which simply introduces random distortions into an incident wave front [3]. Intensity fluctuations develop during free propagation beyond the layer and they may be non-gaussian in both the near field and, for sufficiently small illuminated areas, also in the far field.

The phase-screen model has been used as an approximate theoretical description of a variety of scattering systems ranging from rough surfaces to thermal plumes and has greatly advanced the understanding of propagation through extended media. A number of statistical models for the wave-front distortions have been investigated, mostly based on a joint-gaussian hypothesis for the phase fluctuations [1]. With this assumption, the contrast and correlation properties of the scattered radiation pattern have been calculated by solving Maxwell's equations for a variety of trial phase autocorrelation functions ranging from 'smooth', single scale models of the gaussian-type, to fractal models with power-law spectra [4]. With one notable exception [5], however, it has so far proved to be impossible to evaluate the distribution of intensity fluctuations using this direct approach, although more empirical approaches have met with some success $[6,7]$. This is a serious shortcoming of existing theory in view of the vital role played by the distribution in most noise calculations and also emphasizes the need to investigate the 
sensitivity of phase-screen scattering to the choice of model for the overall phase statistics. Further encouragement for such an investigation has come from some recent experiments $[2,8]$. These have revealed a degree of universality in the statistics of radiation scattered by turbulent media introducing wave-front distortions which are not exactly gaussian distributed [9].

Accordingly, in this paper we present the results of a preliminary theoretical investigation of a simple alternative to the joint-gaussian model. In its most elementary form this is the telegraph-wave phase screen, or a perfectly conducting surface of rectangular grooves, given by the height fluctuations

$$
h(x)=h_{0} T(x)= \pm h_{0},
$$

and having statistical properties governed by the characteristic function

$$
\left\langle\prod_{j} \exp \left[i \lambda_{j} h\left(x_{j}\right)\right]\right\rangle=\left\langle\prod_{j}\left[\cos \lambda_{j} h_{0}+i T\left(x_{j}\right) \sin \lambda_{j} h_{0}\right]\right\rangle .
$$

Various generalizations of this model can be envisaged. For example, a stepped wavefront can be generated by including variable height fluctuations, whilst elementary geometrical optics effects can be introduced by using an integrated version of equation (1). Moreover, the correlation properties determined by equation (2) are closely related to the choice of crossing-distribution for the telegraph wave, so that the phenomenon of 'bunching', presently thought to be important in turbulence scattering, may easily be incorporated into the model [10]. In this first paper, however, we shall restrict our consideration to the random telegraph wave whose zero-crossings are Poisson distributed.

In the next section an exact solution of Maxwell's equations will be obtained for scattering by a perfectly conducting cylindrical surface. Only a brief résumé of the calculation will be presented, a full derivation being reserved for a forthcoming publication.

Apart from a region within a few wavelengths of the surface, this solution reduces to the familiar Huygens-Fresnel formulation of the phase-screen scattering problem. In $\S \S 3$ and 4 , the near-and far-field contrast of the scattered intensity pattern is evaluated for the case of scattering by a random (Poisson) telegraph wave, whilst in $\S 5$ the full distribution of the scattered radiation is calculated for the Fraunhofer configuration. Some conclusions are drawn in $\$ 6$.

\section{The scattering of electromagnetic waves from arbitrary cylindrical infinitely conducting surfaces}

We consider the scattering of electromagnetic waves at an infinitely conducting cylindrical surface (figure 1).

The total field can always be written as a superposition of TE and TM fields which are derived from the Bromwhich potentials, $u$ and $v$ [11]

$$
\left.\begin{array}{c}
\operatorname{TM}\left\{\begin{array} { l } 
{ \mathbf { E } = - \nabla \wedge \mathbf { i } _ { y } \partial u / \partial t , } \\
{ \mathbf { H } = - \mu ^ { - 1 } \nabla \wedge \nabla \wedge \mathbf { i } _ { y } v ; }
\end{array} \quad \operatorname { T E } \left\{\begin{array}{c}
\mathbf{E}=-\varepsilon^{-1} \nabla \wedge \nabla \mathbf{i}_{y} v \\
\mathbf{H}=\nabla \wedge \mathbf{i}_{y} \partial v / \partial t
\end{array},\right.\right. \\
\left(\boldsymbol{\nabla}^{2}-\frac{1}{c^{2}} \frac{\partial^{2}}{\partial t^{2}}\right)\left(\begin{array}{c}
u \\
v
\end{array}\right)=0 .
\end{array}\right\}
$$




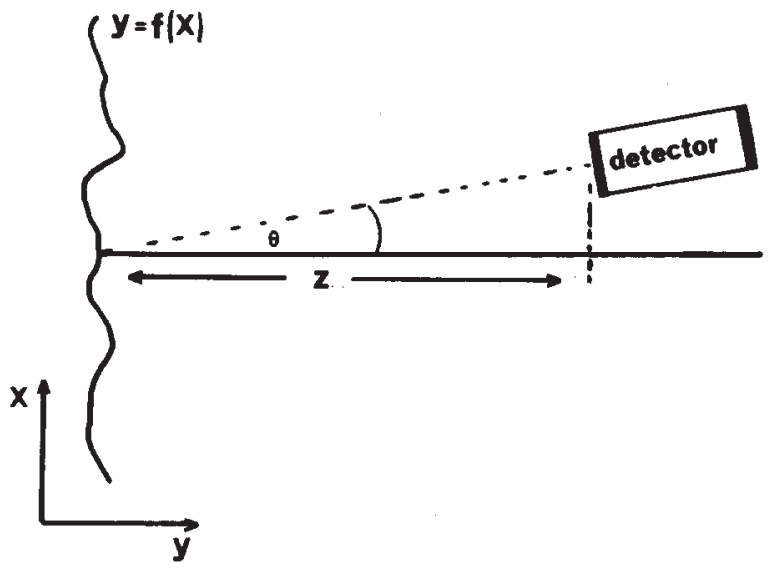

Figure 1. Scattering geometry.

If we restrict ourselves to TM fields which depend only on $x$ and $y$ we obtain

$$
\left.\begin{array}{c}
\mathbf{E}=(i \omega \exp [i \omega t])\left(0,0, \frac{\partial u}{\partial x}\right) ; \\
\mathbf{H}=(-i \omega \exp [-i \omega t])\left(\frac{\partial^{2} u}{\partial x \partial y}, \frac{\partial^{2} u}{\partial x^{2}}, 0\right) ; \\
\left(\frac{\partial^{2}}{\partial x^{2}}+\frac{\partial^{2}}{\partial y^{2}}+\mathrm{k}^{2}\right) u=0 ; \quad \omega=c k
\end{array}\right\}
$$

We will from now on omit the time dependent part ( $-i \omega \exp [i \omega t])$. Suppose that the incoming, field is derived from a potential, $u^{(\mathrm{inc})}$, having the value $u^{(\mathrm{inc})}(x, y=f(x))$ at the boundary $y=f(x)$. The scattered field is then derived from a potential $u^{(\mathrm{sc})}$, satisfying equation (5) the radiation condition at infinity, and the boundary condition

$$
u^{(\mathrm{sc})}(x, f(x))=-u^{(\mathrm{inc})}(x, f(x)) .
$$

The scattering problem is solved on using the following expansion:

$$
\begin{aligned}
& A\left(x^{\prime}\right) \delta\left(x-x^{\prime}\right)+O\left\{\frac{1}{c}\right\}=\int_{-c}^{+c} \exp (i l x) \sin \left(\sqrt{ }\left[k^{2}-l^{2}\right] f(x)\right) \\
& \quad \times \exp \left\{-i l x^{\prime}-\left(i k+h\left(\frac{l}{c}\right)\right) f\left(x^{\prime}\right)\right\} g\left(\frac{l}{c}\right) d l, \quad-\infty<x, x^{\prime}<+\infty,
\end{aligned}
$$

where $A\left(x^{\prime}\right)$ denotes a known function. (We observe that equation (6) is a superposition of planewave solutions of equation (5) at the boundary $y=f(x)$.) The function $g(\lambda)$ is analytic in the domain $|\lambda|<1$ and

$$
\begin{aligned}
& g(\exp [i \phi]) \neq 0, \quad \text { if } \begin{array}{l}
\psi \leqslant \phi \leqslant \pi-\psi, \\
\pi+\psi \leqslant \phi \leqslant \pi-\psi ;
\end{array} \\
& g(\exp [i \phi])=0, \quad \text { if } \begin{array}{l}
-\psi \leqslant \phi \leqslant \psi, \\
\pi-\psi \leqslant \phi \leqslant \pi+\psi ;
\end{array}
\end{aligned}
$$


where

$$
\left.\psi=\operatorname{Liminf}\left\{\phi^{\prime}:\left|\left(x-x^{\prime}\right) \sin \phi\right|\right\rangle\left|\left(f(x)-f\left(x^{\prime}\right)\right) \cos \phi\right|\right\}, \quad-\infty<x, x^{\prime}<+\infty .
$$

The angle $\psi$ has therefore the following property: Suppose we close the contour $-c \leqslant x \leqslant+c$ in either the upper- or lower-part of the complex $l$ plane with a semicircle of radius $R$. Then the main contribution to these integrals is given by the part exp $\left(i l\left(x-x^{\prime}\right)\right)$ of the integrand. This property then leads to the expansion (6) evaluating the right-hand side asymptotically on the semicircles [12].

The function $h(l)$ is analytic in the domain $|l|<1$ and

$$
\operatorname{Re}\{h(\exp [i \phi])\}=|\cos \phi| ; \quad 0 \leqslant \phi \leqslant 2 \pi .
$$

The tedious and lengthy proof of the expansion (6) will be given in a forthcoming paper. We cannot solve the scattering problem on using (6) in its present form because it contains functions which are not the boundary value of an outgoing planewave at the boundary $y=f(x)$. However, using Heine's expansion for an analytic function $g(\lambda)$ :

$$
\begin{aligned}
g(\lambda) & =\sum_{n} a_{n} P_{n}(\lambda) \\
a_{n} & =\left(n+\frac{1}{2}\right) \int_{-1}^{+1} g(\lambda) P_{n}(\lambda) d \lambda,
\end{aligned}
$$

[13], valid within the domain of any ellipse in the complex $\lambda$ plane whose foci are at the points $\lambda= \pm 1$, we derive that:

$$
\begin{aligned}
\exp (i k \cos \phi x+i k \sin \phi y) & =\sum_{n} a_{n} P_{n}(\phi), \\
a_{n} & =\left(n+\frac{1}{2}\right) \int_{-1}^{+1} \exp \left(i k \cos \phi^{\prime} x+i k \sin \phi^{\prime} y\right) P_{n}\left(\phi^{\prime}\right) d \phi^{\prime},
\end{aligned}
$$

with complex $\phi$. Equation (7) shows that every physically non-admittable wave which is not outgoing and/or exploding if we let $y$ tend to infinity, can be approximated arbitrarily closely with a superposition of outgoing planewaves.

Combination of equations (6) and (7) shows that we are able to construct a linear superposition of outgoing planewaves which at the boundary approximate arbitrarily closely a $\delta$ function. The scattering problem is then solved by multiplying this expansion by $-u^{(\text {inc) }}\left(x^{\prime}, f\left(x^{\prime}\right)\right)$ and integrating over $x^{\prime}$ from $-\infty$ to $+\infty$.

Millar [14] has shown that if an approximate solution near and on the boundary of the scattering problem in terms of outgoing waves is obtained, this solution approximates everywhere in the half-space $-\infty<x<+\infty, y>f(x)$ the scattered field in the mean-square sense.

The Huygens-Fresnel approximation of the scattered field can be obtained from equation (6) for surfaces whose extent in the $x$-direction is large compared to a wavelength and whose variation in the $y$-direction over a wave length is small compared to a wavelength. This approximation is also valid for surfaces satisfying the above criteria except for a countable set of points where the variation may be 
infinite [15]. Thus for perfectly conducting cylindrical surfaces of the type (1) the scattered electric field may be written approximately (see figure 1)

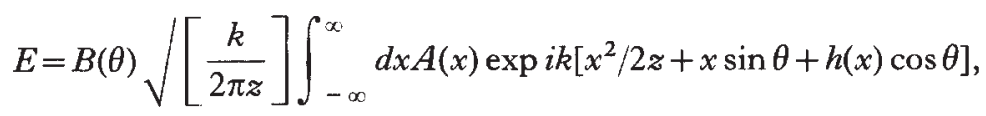

where $B(\theta)$ is a complex angle-dependent factor with constant modulus in the Fresnel region, and $A(x)$ is a real aperture function. In the Fresnel region we set $\theta=0$ in the exponent without loss of generality, whilst in the Fraunhofer region the curvature term, $x^{2} / 2 z$, is neglected.

\section{Intensity fluctuations in the Fresnel region}

It is easily verified that the mean intensity in the Fresnel or near field approximation (8) is constant and may therefore be normalized to unity. The normalized second intensity moment can be expressed in the reduced form

$$
\begin{array}{r}
\frac{\left\langle I^{2}\right\rangle}{\langle I\rangle^{2}}=\frac{k}{2 \pi z} \iint_{-\infty}^{\infty} d x d y \exp \left(\frac{i k x y}{z}\right)\left\langle\operatorname { e x p } i k \left[ h\left(\frac{-x-y}{2}\right)-h\left(\frac{x-y}{2}\right)\right.\right. \\
\left.\left.+h\left(\frac{x+y}{2}\right)-h\left(\frac{y-x}{2}\right)\right]\right\rangle .
\end{array}
$$

Taking advantage of the identity (2), the expectation in the integrand may be written for stationary processes

$$
\begin{array}{r}
\langle\cdot\rangle=\cos ^{4} k h_{0}+\sin ^{2} k h_{0} \cos ^{2} k h_{0}\left[2 g^{(1)}(x)+2 g^{(1)}(y)-g^{(1)}(x+y)-g^{(1)}(x-y)\right] \\
+\sin ^{4} k h_{0} g^{(2)}(x, y),
\end{array}
$$

where

$$
g^{(1)}(x)=\langle T(0) T(x)\rangle ; \quad g^{(2)}(x, y)=\langle T(0) T(x) T(y) T(x+y)\rangle
$$

with

$$
g^{(1)}(x)=g^{(1)}(-x)
$$

and

$$
g^{(2)}(x, y)=g^{(2)}(-x, y)=g^{(2)}(x,-y) .
$$

The symmetry properties can be used to further simplify relation (9):

$$
\frac{\left\langle I^{2}\right\rangle}{\langle I\rangle^{2}}=\frac{4 k}{\pi z} \int_{0}^{\infty} d x \int_{x}^{\infty} d y \cos \left(\frac{k x y}{z}\right)\langle\cdot\rangle .
$$

In order to relate this expression to the properties of the zero-crossings which define the telegraph wave $T(x)$, we introduce the generating function

$$
Q\left(\left\{S_{i}\right\} ;\left\{X_{i}\right\}\right)=\sum_{\{n\}=0}^{\infty} p\left(\left\{n_{i}\right\} ;\left\{X_{i}\right\}\right) \prod_{i}\left(1-S_{i}\right)^{n_{i}}
$$

where $p\left(\left\{n_{i}\right\} ;\left\{X_{i}\right\}\right)$ is the joint distribution of finding $n_{i}$ crossings in the $i$ th consecutive interval $X_{i}$. We have for $x>0$

$$
\langle T(0) T(x)\rangle=\text { probability }(n \text { even in } x) \text {-probability }(n \text { odd in } x \text { ) }
$$


and from (12)

so that

probability $(n$ even in $x)=\frac{1}{2}[Q(2 ; x)+Q(0 ; x)]$,

probability $(n$ odd in $x)=\frac{1}{2}[Q(0 ; x)-Q(2 ; x)]$,

$$
\langle T(0) T(x)\rangle=Q(2 ; x)
$$

An analogous but more lengthy argument leads for $y>x>0$ to

$$
\langle T(0) T(x) T(y) T(x+y)\rangle=Q(2,0,2 ; x, y-x, x) .
$$

Thus (9) may finally be reduced to the form

$$
\frac{\left\langle I^{2}\right\rangle}{\langle I\rangle^{2}}=\cos ^{4} k h_{0}+\frac{4 k}{\pi z} \int_{0}^{\infty} d x \int_{x}^{\infty} d y \cos \left(\frac{k x y}{z}\right) K(x, y),
$$

where

$$
\begin{aligned}
K(x, y)=\sin ^{2} k h_{0} \cos ^{2} k h_{0}[2 Q(2 ; x)+2 Q(2 ; y) & -Q(2 ; x+y)-Q(2 ; y-x)] \\
& +\sin ^{4} k h_{0} Q(2,0,2 ; x, y-x, x) .
\end{aligned}
$$

Equation (15) is quite generally valid for stationary processes; however, we shall now specialize to the case of a random telegraph wave with Poisson distributed crossings, so that [16]

$$
Q(2, x)=\exp (-2 \bar{R} x) \text { and } Q(2,0,2 ; x, y-x, x)=\exp (-4 \bar{R} x)
$$

where $\bar{R}$ is the average crossing rate. Formula (15) can be evaluated exactly for this model to give

$$
\frac{\left\langle I^{2}\right\rangle}{\langle I\rangle^{2}}=1+2 \sin ^{2} k h_{0} \cos ^{2} k h_{0}[1-f(u)-g(u)]+\sin ^{4} k h_{0}\left[1-2 f^{2}(u)-2 g^{2}(u)\right],
$$

where $u=4 \bar{R}^{2} z / k$ and $f$ and $g$ are the auxiliary functions for the Fresnel integrals [17]. The expression (17) is plotted as a function of $\sqrt{ }[2 u / \pi]$ for various values of $k h_{0}$ in the first quadrant in figure 2 . As expected these scintillation plots show no focusing

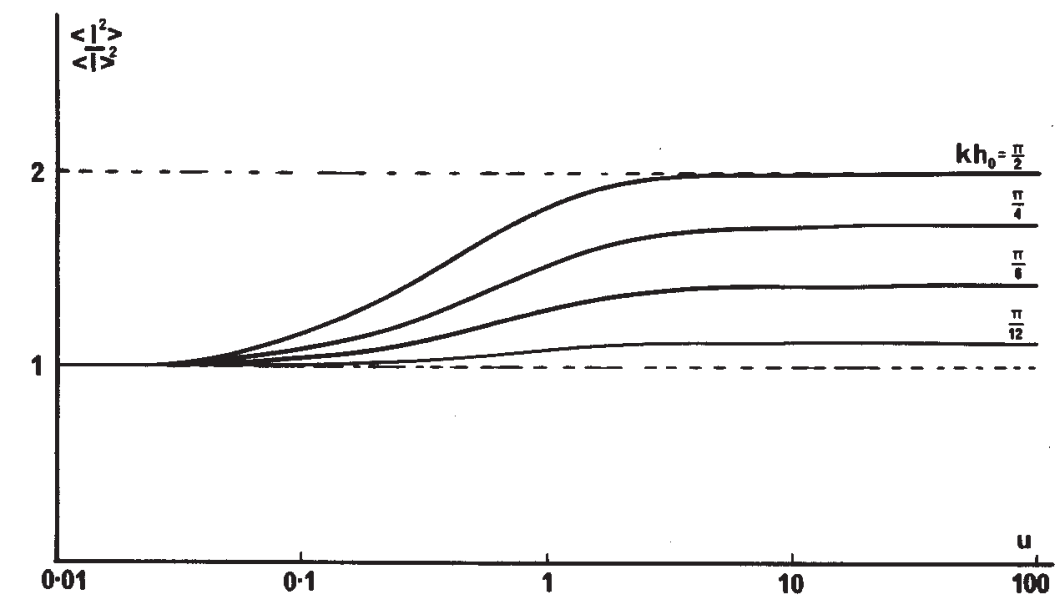

Figure 2. Second moment of the intensity fluctuation distribution in the Fresnel region as a function of distance for various surface heights. 
effects, being monotonically increasing functions of the single parameter $u$. The special case $k h_{0}=\pi / 2$ coincides with the result obtained for scattering by a gaussian phase screen with linear correlation function (the so-called Brownian fractal) $[4,11]$. It is interesting that the same result is also obtained for scattering by a randomly stepped surface when the heights of the steps are odd multiples of one fourth the radiation wavelength, i.e. if instead of (1) we had adopted the model $h(x)=n(x) \lambda / 4$ where $n(x)$ is an odd integer. For large $u$ the scattered field is a circular complex gaussian process. Saturation of the second moment at values below the gaussian speckle value of 2 in this limit (figure 2) reflects the presence of a significant unscattered component when $h_{0}$ is not an odd multiple of half the wavelength. A Rice distribution of intensity fluctuations is obtained in this case [18].

\section{Intensity fluctuations in the Fraunhofer region}

As our starting point for a far-field calculation we use a normalized version of equation (8) with hard aperture of dimension $L$ :

$$
\begin{aligned}
E & =\frac{1}{L} \int_{-L / 2}^{L / 2} \exp i k\left(x \sin \theta+h_{0} T(x) \cos \theta\right) d x \\
& =\cos \left(k h_{0} \cos \theta\right)+\frac{i}{L} \int_{-L / 2}^{L / 2} T(x) \sin \left(k h_{0} \cos \theta\right) \exp (i k x \sin \theta) d x .
\end{aligned}
$$

Thus the scattered field may be expressed as the sum of a constant or unscattered component and a fluctuating part of zero mean. Calculation of the mean and meansquare intensity from this formula for the case of a random telegraph wave with Poisson-distributed crossings is straightforward but tedious and is briefly outlined in the Appendix. The results for arbitrary scattering angle $\theta$ are lengthy but in the forward direction the formulae reduce to

$$
\begin{aligned}
\langle I\rangle= & \cos ^{2} k h_{0}+\sin ^{2} k h_{0}\left[1 / \bar{N}-1 / 2 \bar{N}^{2}+\exp (-2 \bar{N}) / 2 \bar{N}^{2}\right], \\
\left\langle I^{2}\right\rangle= & \cos ^{4} k h_{0}+\frac{\sin ^{2} k h_{0} \cos ^{2} k h_{0}}{\bar{N}^{2}}[2 \bar{N}+\exp (-2 \bar{N})-1] \\
& +\frac{\sin ^{4} k h_{0}}{\bar{N}^{2}}\left[3 \bar{N}^{2}-6 \bar{N}+\frac{9}{2}-\left(3 \bar{N}+\frac{9}{2}\right) \exp (-2 \bar{N})\right],
\end{aligned}
$$

where $\bar{N}=\bar{R} L$. It is clear from these formulae that the constant unscattered contribution always dominates the statistics in the limit $\bar{N} \rightarrow \infty$ unless $h_{0}$ is an odd multiple of $\lambda / 4$, so that with this exception the contrast of the pattern vanishes in this limit. The contrast also vanishes, as expected, in the limit $\bar{N} \rightarrow 0$, when the surface grooves are much larger than the aperture so that no scattering occurs. In the special case when $k h_{0}=(2 n+1) \pi / 2$, the mean-square intensity saturates at a value of 3 for large $\bar{N}$. This is a consequence of the fact that the first term in equation (18) vanishes whilst the second is simply a (one-dimensional) gaussian in the large step number limit. The above mentioned features are illustrated in plots of the normalized second intensity moment shown in figure 3 .

The off-axis behaviour of the intensity is complicated by the double periodicity associated with the height $h_{0}$ and aperture size $L$. For angles $\theta$ greater than the central 'speckle' $\sin ^{-1}(k L)^{-1}$ the unscattered component becomes progressively less 


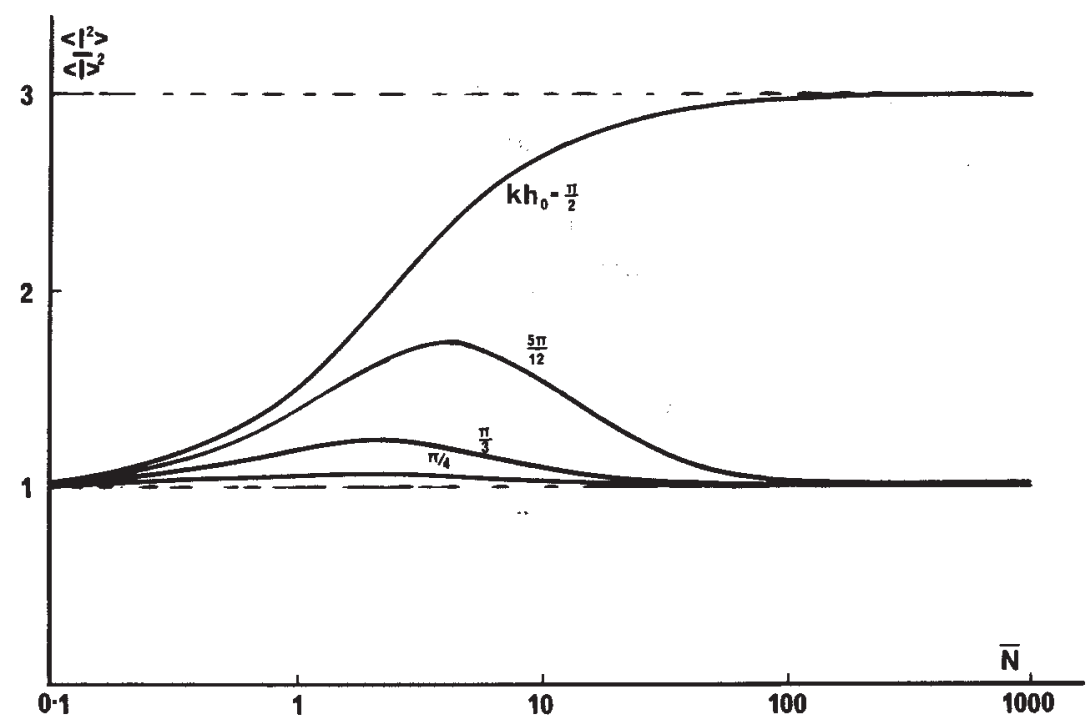

Figure 3. Second intensity moment in the far-field specular direction as a function of aperture size for various surface heights.

important and the contrast of the pattern approaches the value of unity for large $\bar{N}$, corresponding to a complex gaussian scattered field.

\section{Far-field intensity fluctuation distribution}

In the forward direction equation (18) reduces to

$$
E=\cos k h_{0}+\sin k h_{0} \frac{i}{L} \int_{-L / 2}^{L / 2} T(x) d x,
$$

so that the intensity is given by

$$
I=\cos ^{2} k h_{0}+\sin ^{2} k h_{0}\left[\frac{1}{L} \int_{-L / 2}^{L / 2} T(x) d x\right]^{2} .
$$

The characteristic function for the fluctuating component in these expressions,

$$
C(\lambda)=\left\langle\exp \frac{i \lambda}{L} \int_{-L / 2}^{L / 2} T(x) d x\right\rangle,
$$

has been evaluated exactly by previous authors in a different context and may be written in the form [19],

$$
C(\lambda)=\exp (-\bar{N})\left\{\cosh \sqrt{[}\left[\bar{N}^{2}-\lambda^{2}\right]+\frac{\bar{N}}{\sqrt{ }\left[\bar{N}^{2}-\lambda^{2}\right]} \sinh \sqrt{ }\left[\bar{N}^{2}-\lambda^{2}\right]\right\} .
$$

The distribution of intensity can now be calculated using (22), and the following result is obtained for the regime $\cos ^{2} k h_{0}<I<1$ :

$P(I)=\frac{\bar{N} \exp (-\bar{N}) \sin k h_{0}}{\sqrt{ }\left[I-\cos ^{2} k h_{0}\right]}\left\{I_{0}\left(\bar{N} \sqrt{ }[1-I] / \sin k h_{0}\right)+\frac{I_{1}\left(\bar{N} \sqrt{ }[1-I] / \sin k h_{0}\right)}{\sqrt{ }[1-I] / \sin k h_{0}}\right\}$,

where $I_{0}$ and $I_{1}$ are modified Bessel functions of the first kind. For $I<\cos ^{2} k h_{0}$ and $I>1$ the distribution vanishes as expected from the form of equation (22). When the 
surface height is an odd multiple of quarter wavelengths $\left(k h_{0}=(2 n+1) \pi / 2\right.$, formula (25) reduces to the simpler expression

$$
P(I)=\frac{\bar{N} \exp (-\bar{N})}{\sqrt{I}}\left\{I_{0}(\bar{N} \sqrt{ }[1-I])+\frac{I_{1}(\bar{N} \sqrt{ }[1-I])}{\sqrt{ }[1-I]}\right\}, \quad 0<I<1,
$$

which is plotted for various values of $\bar{N}$ in figure 4 . The moments of this distribution are given by

$$
\left\langle I^{n}\right\rangle=\left(\frac{2}{\bar{N}}\right)^{n-1 / 2} \Gamma\left(n+\frac{1}{2}\right) \exp (-\bar{N})\left[I_{n+1 / 2}(\bar{N})+I_{n-1 / 2}(\bar{N})\right]
$$

and reduce after normalization to $2^{n} \Gamma\left(n+\frac{1}{2}\right) / \sqrt{ } \pi$, when $\bar{N}$ is large, corresponding to a gaussian distribution of the scattered field. As discussed earlier, this is a consequence of the one-dimensional random walk implicit in equations (18) and (21).

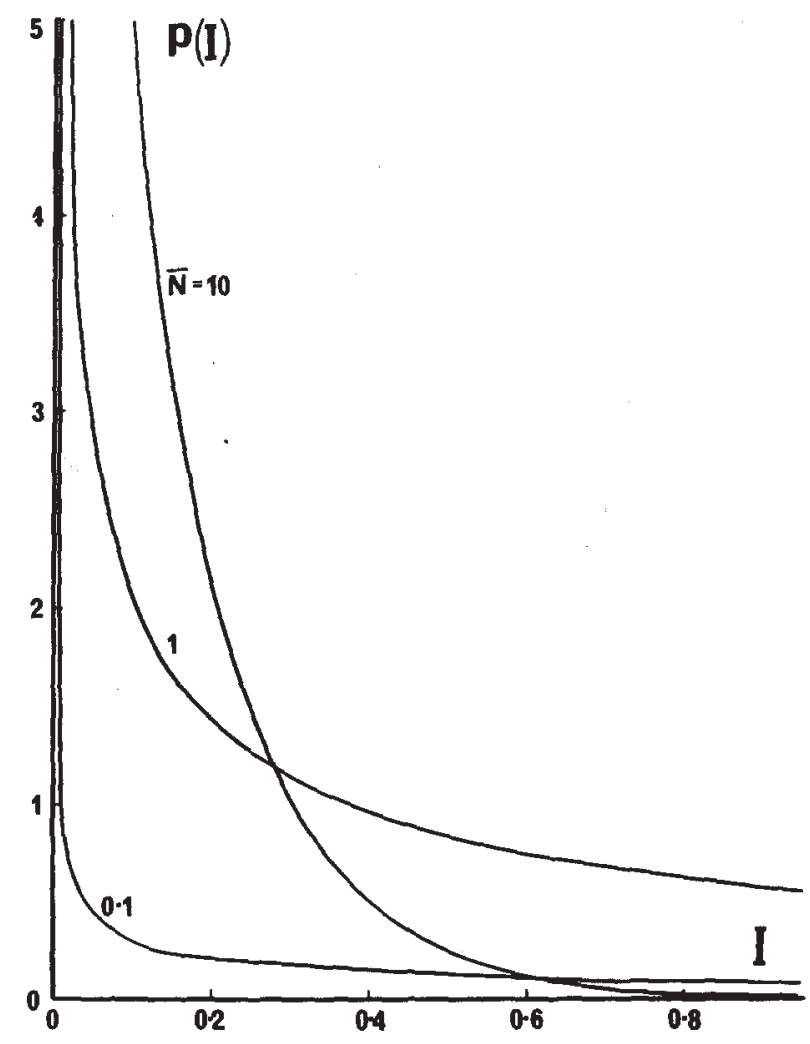

Figure 4. Distribution of intensity fluctations in the far-field specular direction as a function of aperture size for $k h_{0}=\pi / 2$.

Results (26) and (27) represent the extreme diffuse or incoherent scattering limit. As the surface-height fluctuations approach multiples of half the wavelength $\left(k h_{0}=n \pi\right)$ the unscattered component in equation (21) dominates, formula (25) reduces to a delta-function and no-intensity fluctuations occur in the forward direction $(\theta=0)$. The situation off-axis is evidently more complicated and the existence of an exact solution of the problem seems unlikely. 


\section{Discussion}

It is interesting to compare the results of the present calculations with those obtained for scattering by a gaussian random surface with linear correlation function (the so-called 'Brownian' fractal), [4, 18, 20]. In the Fresnel region [18], expressions for the contrast of the scattered intensity pattern coincide when the groove height is an odd multiple of one fourth the wavelength. Indeed it appears that a wider class of step-like surfaces can generate Fresnel region intensity patterns with the same contrast as that produced when radiation is scattered by a Brownian fractal. The farfield intensity patterns generated by the two models are, however, quite different in character, particularly in the forward scattering direction [20]. Both models are then characterized by a single 'strength' parameter. When this is large, the surface of random rectangular grooves gives a second normalized intensity moment ranging from unity to three depending on the strength of the unscattered contribution to the field (governed by the value of $k h_{0}$ in equation (20)), whilst the gaussian-linear correlation function model gives the usual fully developed speckle pattern result of two corresponding to a complex or two-dimensional gaussian field distribution (equation (A4) and figure 3 of [20]).

In conclusion it is evident that surfaces of rectangular grooves form an interesting class of random scattering worthy of further investigation. They provide a relatively simple alternative to the well tried class of gaussian models and may provide a better description of certain types of scattering system [10]. For example, it has been found that a layer of mixing, weakly diffusing fluids (e.g. brine/pure water) scatters laser light to give scintillation plots akin to figure 2-apparently due to the relatively sharp boundaries which persist between regions of different refractive index. It is perhaps also worth pointing out that the results of this paper represent an original contribution to the literature on diffraction gratings [21], equation (18) being interpreted as representing the far field of either a random phase or random amplitude grating.

\section{Acknowledgment}

This work was supported by NATO Research Grant No. 061-81.

\section{Appendix}

From equation (18) the mean-scattered intensity can be expressed in the form

$$
\langle I\rangle=\frac{1}{L^{2}} \int_{-L / 2}^{L / 2} d x \int_{-L / 2}^{L / 2} d x^{\prime} \exp i k\left(x-x^{\prime}\right) \sin \theta\left[\cos ^{2} \phi+\sin ^{2} \phi Q\left(2,\left|x-x^{\prime}\right|\right)\right],
$$

where $Q$ is given by equation (16) and $\phi=k h_{0} \cos \theta$. Equation (A 1) may be evaluated to give the result

$$
\langle I\rangle=\frac{2 \cos ^{2} \phi(1-\cos \psi)}{\psi^{2}}+2 \sin ^{2} \phi \operatorname{Re}\left\{\alpha-\alpha^{2}+\gamma \alpha^{2}\right\}
$$

where

$$
\psi=k L \sin \theta, \quad \alpha=(2 \vec{N}-i \psi)^{-1} \quad \text { and } \quad \gamma=\exp \left(-\alpha^{-1}\right)
$$


The second moment of the intensity fluctuation distribution is more complicated and may be expressed in the form

$$
\begin{aligned}
\left\langle I^{2}\right\rangle= & \frac{1}{L^{4}} \int_{-L / 2}^{L / 2} d x \int_{-L / 2}^{L / 2} d x^{\prime} \int_{-L / 2}^{L / 2} d x_{1} \int_{-L / 2}^{L / 2} d x_{1}^{\prime} \exp \left\{i k\left[\left(x-x^{\prime}\right)+\left(x_{1}-x_{1}^{\prime}\right)\right] \sin \theta\right\} \\
& \times\left[\cos ^{4} \phi+\sin ^{2} \phi \cos ^{2} \phi\left\{\left\langle T(x) T\left(x^{\prime}\right)\right\rangle+\left\langle T(x) T\left(x_{1}^{\prime}\right)\right\rangle+\left\langle T\left(x^{\prime}\right) T\left(x_{1}\right)\right\rangle\right.\right. \\
& \left.+\left\langle T\left(x_{1}\right) T\left(x_{1}^{\prime}\right)\right\rangle-\left\langle T(x) T\left(x_{1}\right)\right\rangle-\left\langle T\left(x^{\prime}\right) T\left(x_{1}^{\prime}\right)\right\rangle\right\} \\
& \left.+\sin ^{4} \phi\left\langle T(x) T\left(x^{\prime}\right) T\left(x_{1}\right) T\left(x_{1}^{\prime}\right)\right\rangle\right] .
\end{aligned}
$$

The first term in the square brackets is just

$$
4 \cos ^{4} \phi(1-\cos \psi)^{2} / \psi^{4} .
$$

The second term may be evaluated with the help of equations (13) and (16) to give $\frac{8 \sin ^{2} \phi \cos ^{2} \phi(1-\cos \psi)}{\psi^{2}}\left[\cos \psi-\exp (-2 \bar{N})-\frac{2 \bar{N}}{\psi} \sin \psi+2 \operatorname{Re}\left\{\alpha+\alpha^{2} \gamma-\alpha^{2}\right\}\right]$.

The final term is evaluated using the relation $\left(x_{1}<x_{2}<x_{3}<x_{4}\right)$

$$
\left\langle T\left(x_{1}\right) T\left(x_{2}\right) T\left(x_{3}\right) T\left(x_{4}\right)\right\rangle=\exp \left[-2 \bar{R}\left(x_{1}-x_{2}+x_{3}-x_{4}\right)\right],
$$

which is the most general form of equation (14) for a Poisson distribution of crossings. The calculation is straightforward, though lengthy, and the final result is

$$
\begin{aligned}
4 \sin ^{4} \phi \operatorname{Re}\left\{\begin{array}{l}
\alpha^{2}-4 \alpha^{3}+6 \alpha^{4}+|\alpha|^{2}-2 \alpha^{2} \alpha^{*}+2 \alpha^{3} \alpha^{*}-2 \alpha^{*} \alpha^{2}+4|\alpha|^{4} \\
-\gamma\left[2 \alpha^{3}+6 \alpha^{4}+4 \alpha^{3} \alpha^{*}+4|\alpha|^{4}+2 \alpha^{2} \alpha^{*}\right]
\end{array}\right. \\
\quad+\frac{1}{i \psi}\left[2 \alpha \alpha^{* 2}-\alpha^{2}+\alpha^{3}-\alpha^{2} \alpha^{*}-\alpha^{*}-\gamma\left(\alpha^{* 2} \alpha+\alpha^{3}+\alpha^{2} \alpha^{*}\right)\right. \\
\left.\left.-\exp (i \psi) \alpha \alpha^{* 2}+\alpha^{*} \gamma^{*}\right]+\frac{|\alpha|^{2}}{2 \psi^{2}}[1-\exp (i \psi)]\right\} .
\end{aligned}
$$

The normalized second intensity moment is thus given by

$$
\frac{\left\langle I^{2}\right\rangle}{\langle I\rangle^{2}}=\frac{(\mathrm{A} 4)+(\mathrm{A} 5)+(\mathrm{A} 7)}{(\mathrm{A} 2)^{2}} \text {. }
$$

Les résultats d'une étude théorique préliminaire des propriétés statistiques d'un rayonnement électromagnétique diffusé par une surface striée d'une manière aléatoire sont présentés. Le contraste de la répartition d'intensité est calculé à la fois pour des géométries en champ proche et lointain, et on montre que la densité de probabilité des fluctuations d'intensité peut être obtenue avec exactitude dans la direction spéculaire en champ lointain.

Es werden Ergebnisse einer vorläufigen theoretischen Untersuchung der statistischen Eigenschaften von elektromagnetischer Strahlung präsentiert, die an einer zufälig gefurchten Oberfläche gestreut wird. Der Kontrast der Intensitätsverteilung wird sowohl für das Nahfeld als auch für das Fernfeld berechnet und es wird gezeigt, daß die vollständige Wahrscheinlichkeitsdichte der Intensitätsfluktuationen für die Spiegelungsrichtung im Fernfeld exakt bestimmbar ist. 


\section{References}

[1] Jakemax, E., 1980, Applications of Speckle Phenomena, edited by W. H. Carter, SPIE Proceedings, Vol. 243 (Bellingham: Society of Photo-optical Instrumentation Engineers), 9.

[2] Jakeman, E., and Pusey, P. N., 1980, Inverse Scattering Problems in Optics, edited by H. P. Baltes, Topics in Current Physics, Vol. 20 (Berlin: Springer-Verlag), 73.

[3] Zardecki, A., 1978, Inverse Source Problems in Optics, edited by H. P. Baltes, Topics in Current Physics, Vol. 9 (Berlin: Springer-Verlag), 155.

[4] Berry, M. V., 1979, J. Phys. A, 12, 781.

[5] Jakemax, E., 1982, J. Phys. A, 15, L55.

[6] Jakemax, E., and Pusey, P. N., 1978, Phys. Rev. Lett., 40, 546.

[7] Hoenders, B. J., Jakeman, E., Baltes, H. P., and Steinle, B., 1979, Optica Acta, 26, 1307.

[8] Jakeman, E., 1980, J. Phys. A, 13, 31.

[9] Sснӓтzед, K., 1980, Proceedings of the Fourth International Conference on Photon Correlation Techniques in Fluid Mechanics (Stanford University: Joint Institute for Aeronautics and Acoustics).

[10] Jakeman, E., 1981, Optica Acta, 28, 435.

[11] Вromwhich, T. J. I'A., 1919, Phil. Mag., 38, 143.

[12] Hoenders, B. J., 1978, J. Phys. A, 11, 1815.

[13] Whittaker, E. T., and Watson, G. N., 1963, A Course on Modern Analysis (Cambridge University Press).

[14] Millar, R. F., 1973, Radio Sci., 8, 785.

[15] van Kampen, N. G., 1949, Physica, 14, 575.

[16] Davenport, W. B., and Root, W. L., 1958, Random Signals and Noise (New York: McGraw-Hill), Chap. 4.

[17] Arbramowitz, M., and Stegun, I., 1965, Handbook of Mathematical Functions (New York: Dover).

[18] Jakeman, E., and McWhirter, J. G., 1977, J. Phys. A, 10, 1599.

[19] See, for example, Morrison, J. A., and McKenna, J., 1973, Proceedings of the SIAMAMS, Vol. 6 (Providence, R.I.: American Mathematical Society), p. 97.

[20] Walker, J. G., and Jakeman, E., 1982, Optica Acta, 29, 313.

[21] Petit, R. (editor), 1980, Electromagnetic Theory of Gratings, Topics in Current Physics, Vol. 22 (Berlin: Springer-Verlag). 